\title{
Integrative multi-omics identifies high risk multiple myeloma subgroup associated with significant DNA loss and dysregulated DNA repair and cell cycle pathways
}

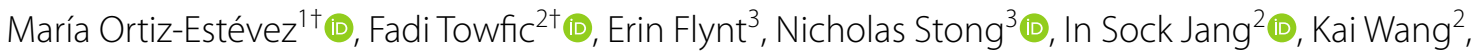
Matthew W. B. Trotter ${ }^{1}$ and Anjan Thakurta ${ }^{3 *}$

\begin{abstract}
Background: Despite significant therapeutic advances in improving lives of multiple myeloma (MM) patients, it remains mostly incurable, with patients ultimately becoming refractory to therapies. MM is a genetically heterogeneous disease and therapeutic resistance is driven by a complex interplay of disease pathobiology and mechanisms of drug resistance. We applied a multi-omics strategy using tumor-derived gene expression, single nucleotide variant, copy number variant, and structural variant profiles to investigate molecular subgroups in 514 newly diagnosed MM (NDMM) samples and identified 12 molecularly defined MM subgroups (MDMS1-12) with distinct genomic and transcriptomic features.
\end{abstract}

Results: Our integrative approach let us identify NDMM subgroups with transversal profiles to previously described ones, based on single data types, which shows the impact of this approach for disease stratification. One key novel subgroup is our MDMS8, associated with poor clinical outcome [median overall survival, 38 months (global log-rank $p$-value $\left.<1 \times 10^{-6}\right)$ ], which uniquely presents a broad genomic loss ( $>9 \%$ of entire genome, $t$-test $p$ value $<1 \mathrm{e}-5$ ) driving dysregulation of various transcriptional programs affecting DNA repair and cell cycle/mitotic processes. This subgroup was validated on multiple independent datasets, and a master regulator analyses identified transcription factors controlling MDMS8 transcriptomic profile, including CKS1B and PRKDC among others, which are regulators of the DNA repair and cell cycle pathways.

Conclusion: Using multi-omics unsupervised clustering we were able to discover a new high-risk multiple myeloma patient segment. This high-risk group presents diverse previously known genetic markers, but also a new characteristic defined by accumulation of genomic loss which seems to drive transcriptional dysregulation of cell cycle, DNA repair and DNA damage. Finally, our work identified various master regulators, including E2F2 and CKS1B as the genes controlling these key biological pathways.

*Correspondence: anjan.thakurta@bms.com

†María Ortiz-Estévez and Fadi Towfic contributed equally to this work and are co-lead authors

${ }^{3}$ Bristol Myers Squibb, 181 Passaic Ave, Summit, NJ 07901, USA

Full list of author information is available at the end of the article

\section{Introduction}

Multiple Myeloma (MM) patients have complex genetic heterogeneity in the tumor that includes structural variants (SVs) such as immunoglobulin heavy chain $(I g H)$ translocations, single nucleotide variants (SNVs) in oncogenes and tumor suppressor genes, and genomic/ chromosomal copy number variants (CNVs), as well as 
transcriptomic changes [1, 2]. A comprehensive molecular classification of the disease based on all these types of data may shed light into how the combinations of these genetic and transcriptomic features define or contribute to intra-tumoral heterogeneity, therapeutic response and/or resistance and eventual relapse.

The MM community has devoted significant effort toward identifying molecular genetic features to diagnose MM patients, especially focused on patients with poor prognosis. For this reason, they have relied upon supervised analyses to identify molecular features associated with poor clinical outcome that may not necessarily identify biological sub-types of disease, nor be the features driving aggressive biology of the tumor. Various signatures have been previously proposed to identify high-risk patients, including UAMS70/80/17 [3], EMC92 [4], IFM15 [5], chromosome instability signature [6], centrosome index signature [7] and proliferation index [8]. Some of these signatures were combined with disease stages [9] or expression of long intergenic non-coding RNAs [10] to improve their prognostic utility. Recently, we identified high-risk disease subgroups based on DNA features combining amp1q $(\mathrm{CNV}=4$ or more) plus International Staging System 3 (ISS) or biallelic inactivation of TP53 (deletion and mutation) [11]; and clonal status of del17p (high-risk del17p) [12]. To date, some genomic biomarkers including del17p, gain1q, $t(4 ; 14)$ or $t(14 ; 16)$, and mutations in TP53, in combination with clinical characteristics have been used in the clinic or clinical trials for prognosis $[13,14]$.

Previous efforts to stratify MM based on gene expression (GE) data identified 7 molecular subgroups with distinct transcriptomic profiles [15-17]. Some of these subgroups were linked to genomic abnormalities (including translocations (SVs) or hyperdiploidy (HY)), while others such as the proliferative group (PR) apparently was driven mainly by transcriptional pathways [15]. More recently, Laganà et al. identified gene modules, which were subsequently associated with genomic and clinical features [17]. Mutational signatures that are independent of previously defined prognostic markers have also been used to stratify MM patients [18] and stratification of MM patients based on CNVs has demonstrated some association with outcome [19].

Integrative clustering analyses across multiple data types from large, well annotated datasets, have identified novel biological subgroups in solid tumors and acute myeloid leukemia [20-23]; showing the impact of data integration in disease stratification. Such an analysis, however, is yet to be reported in MM. As part of the Myeloma Genome Project (MGP) [19], here we present a large-scale multi-omics analysis of newly diagnosed MM (NDMM).
Our work identified 12 disease subgroups using an integrative multi-omics approach combining GE, SV, $\mathrm{CNV}$, and SNV features (Fig. 1A), where clinical covariates, such as outcome data, were not included to define genomic subgroups independently from known clinical features. We further explored the molecular features and clinical associations of the 12 biological subsets and focused on a subgroup (MDMS8) which showed the worst prognosis across the entire patient cohort (Fig. 1B). MDMS8 main characteristic is a significant $(>8 \%)$ genomic loss associated with dysregulated DNA repair and cell cycle/mitotic related transcriptional programs. The integrative nature of MDMS8 comes up on its transversal profile to specific known biomarkers of high risk (including 1q amplification, del17p and $\mathrm{t}(4 ; 14)$ (Fig. 2 and Additional file 1: Figs. S4 and S5A-E), and to patient subgroups previously defined based only on gene expression [such as the proliferative, the MMSET and the MAF subgroups (15-17)] (Fig. 6). Master regulator analysis [24, 25] identified 7 genes controlling MDMS8 transcriptional program, including E2F2, CKS1B and PRKDC, which seem to control dysregulation of DNA repair and cell cycle pathways putatively for sustaining the genome loss. We further validated MDMS8 in independent NDMM and relapsed/refractory MM (RRMM) datasets demonstrating the reproducible persistence and prevalence of this segment across patient cohorts.

\section{Results}

Integrative clustering analysis identifies twelve molecularly defined disease subgroups in myeloma

We analyzed genomic and transcriptomic data from 514 NDMM patients enrolled in the Multiple Myeloma Research Foundation (MMRF) CoMMpass study (NCT0145429, version IA17). The subset of the samples selected was based on the intersection of the various datasets (GE, CNVs, SNVs, SVs and clinical information), and patient characteristics are presented in Additional file 1: Table S1. Demographics, clinical data, treatment information and data processing steps have been published previously $[11,19]$.

Two alternative multi-omics integrative analysis methods were applied to the complete dataset: iCluster $+[26]$ and Cluster of Clusters Analysis (COCA) [27]. Each clustering method was run one thousand times with resampling of features and samples to ensure robustness (Additional file 1: Fig. S1). While iCluster + defines clusters based on integrated, simultaneous analysis across the data types; COCA uses a two-step analysis, first clustering on each single data type and then grouping the results into a final set of clusters. Results of the two clustering methods overlapped but were not identical (Additional file 1: Table S2). In our dataset, iCluster+identified 12 


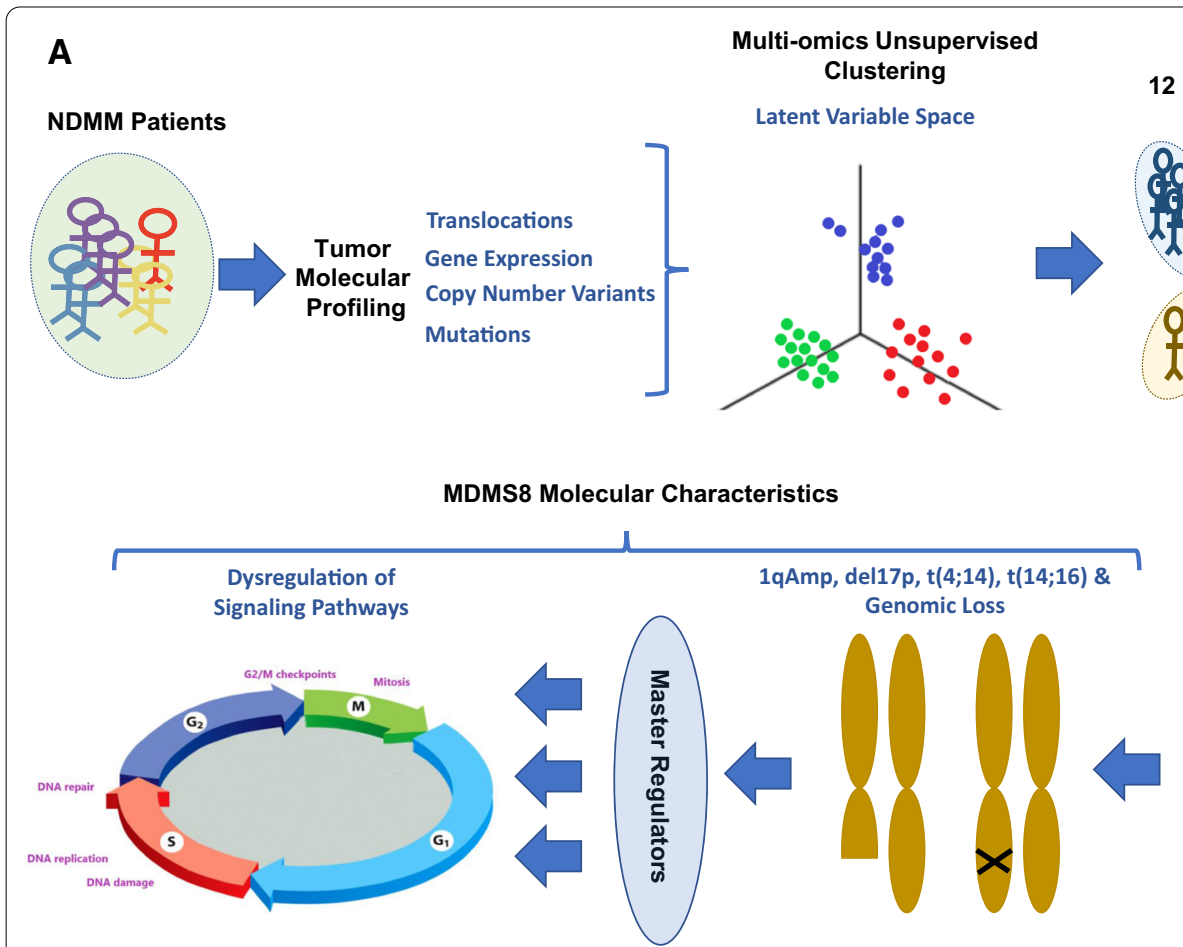

B Copy Number Variants
Gene Expression
12 Molecularly Defined Myeloma Segments (MDMS1-12)

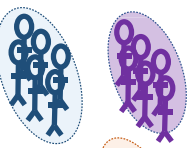

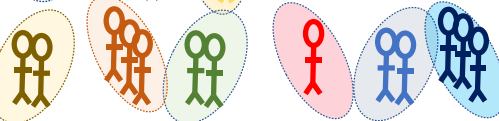

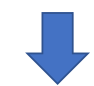

MDMS8 is HR

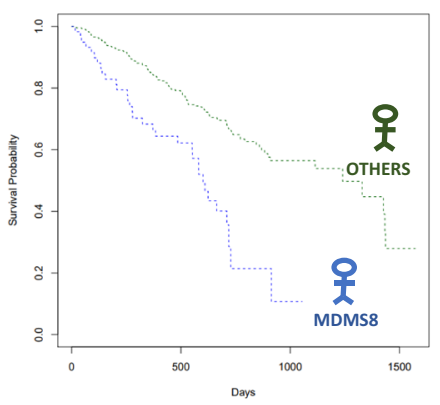

Single Nucleotide Variants

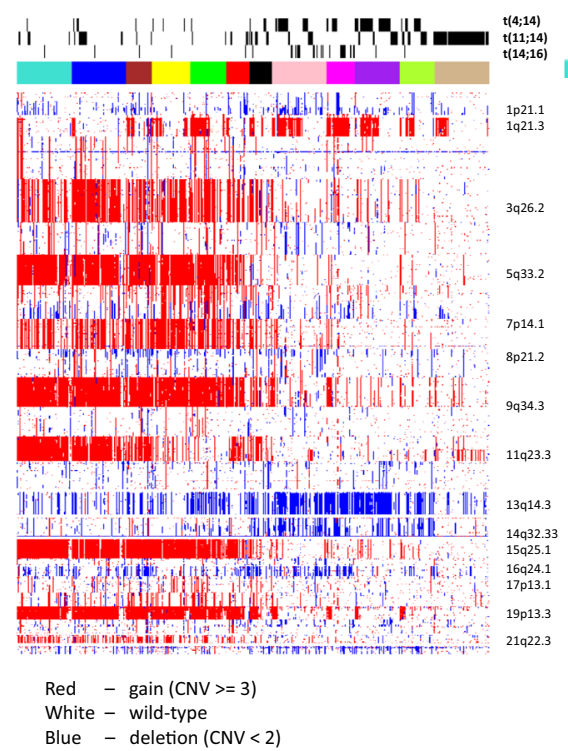

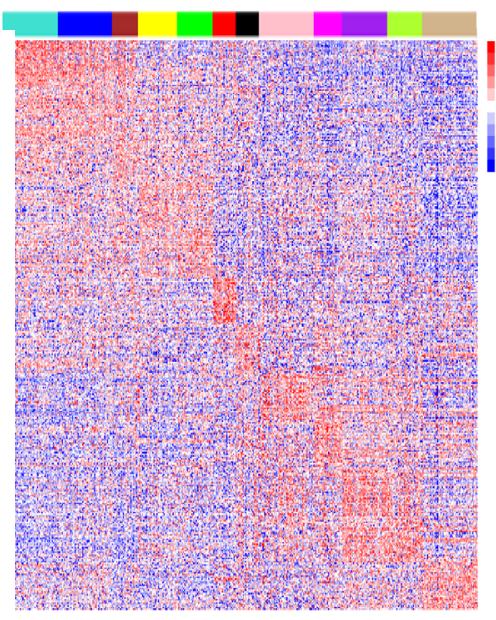

Blue - deletion $(\mathrm{CNV}<2)$

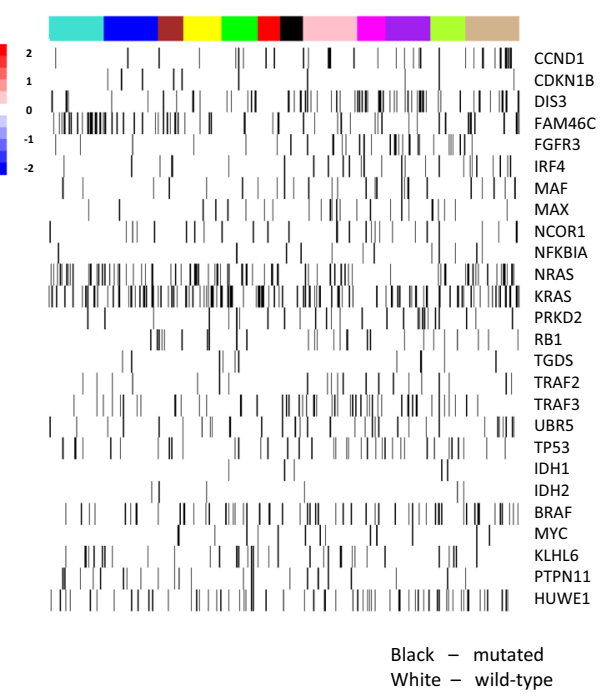

Fig. 1 Twelve multiple myeloma subgroups identified by integrative clustering. A Figure representing a visual summary of the work presented in the paper. From NDMM molecular profiles to identification of HR patient segment by multi-omics unsupervised clustering and its main characteristics including genomic loss, master regulators and DNA repair and cell cycle dysregulation. B Heatmap showing molecular characteristics of the molecularly defined myeloma subgroups (MDMS 1-12): Left panel shows copy number variants with structural variants added as tracks above; middle panel shows gene expression (top 30 over-expressed genes per MDMS without replication); and right panel shows single nucleotide variants (black band denotes mutation, white band denotes wild-type sequence) 


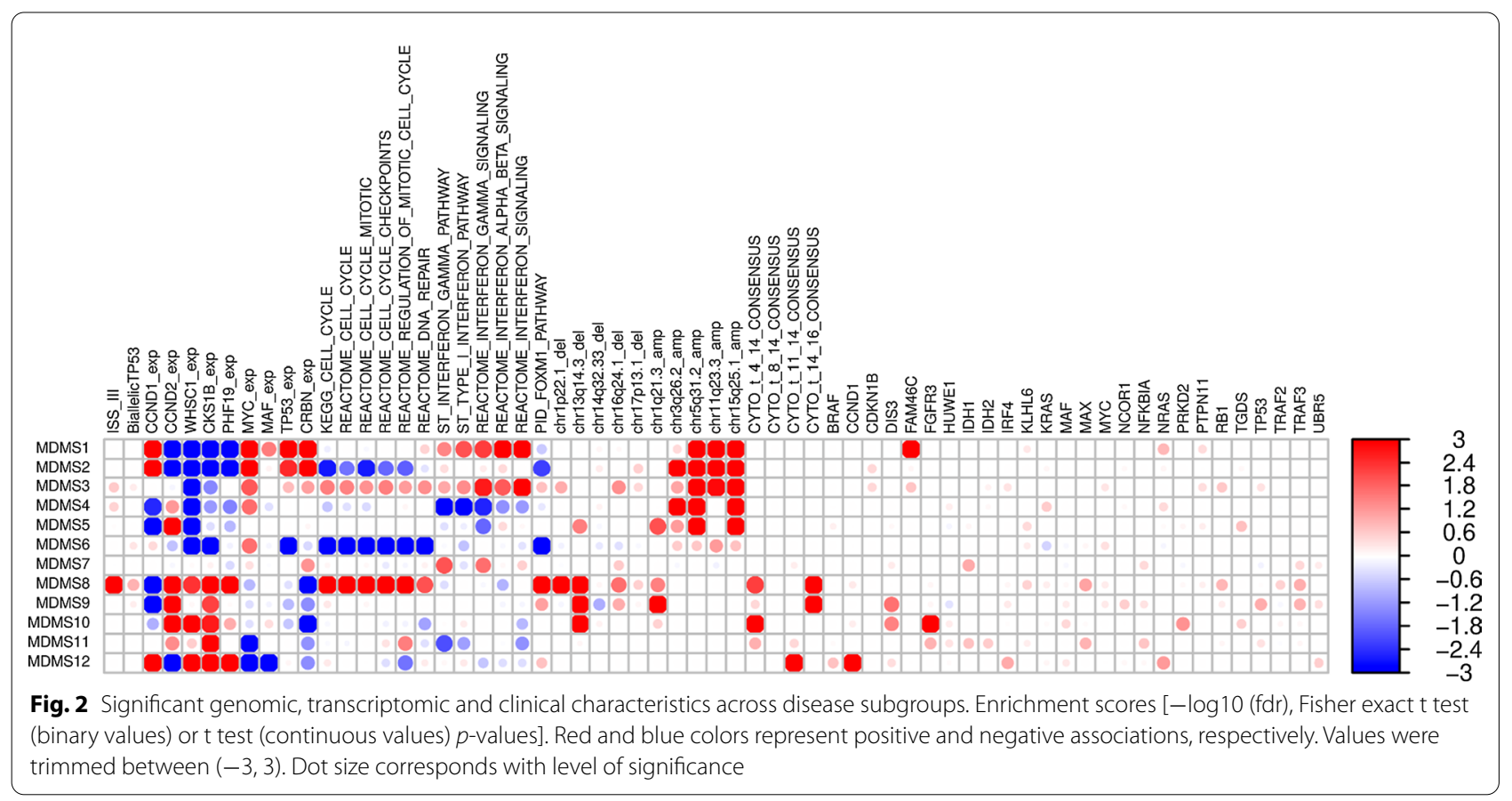

subgroups (in $>40 \%$ of the iterations, followed by 11 clusters selected $<30 \%$ ) compared to 14 subgroups $(>30 \%$ of the iterations, followed by 12 clusters selected $<20 \%$ ) identified by COCA. Consensus across iterations, defined by prevalence of same samples being clustered together, was higher in iCluster $+(>70 \%$ iCluster $+v s<65 \%$ COCA) thus, the iCluster + output was selected for further analysis.

Twelve molecularly defined MM subgroups (MDMS) were identified by iCluster + (Additional file 1: File 2), with sizes ranging from 5 to $12 \%$ of the total cohort of 514 (Fig. 1B and Additional file 1: Fig. S2). These included six HY subgroups (MDMS1-6), characterized by gains ( $\mathrm{CNV}=3$ or more) of chromosomes 3 , 5, 9, 15 and 19, and six non-HY subgroups (MDMS712) (Figs. 1B and 2; Additional file 1: Table S3). Within the HY group, MDMS1-2-3 share several molecular characteristics, including gain of Chr11 (gain11) and over-expression of PAPD7. MDMS1 is differentiated from MDMS3 and MDMS5 by deletion of 8p22.1 (del8p22.1), mutation of $R B 1$, over-expression of $N S D H L$ and up-regulated cell cycle and checkpoints signaling pathways. MDMS2 shows a deep down-regulation of cell cycle related pathways, and this characteristic is shared with MDMS6. MDMS3 is enriched in FAM46C and NRAS mutation and up-regulation of the interferon pathway. MDMS4 and MDMS5 have no gain of Chr11, but MDMS5 only is enriched in gain of Chr3 and has significant del13q and mutations in
ARID2, EGR1 and NF1 genes. MDMS6 is defined by gain20q11, gain11q23.3, down-regulation of MED11, and down-regulation of DNA repair, cell cycle and checkpoints pathways (Figs. 1B and 2; Additional file 1: Table S3).

Among the non-HY subgroups, MDMS7, MDMS11 and MDMS12 are significantly associated with $t(11 ; 14)$ (Figs. 1B and 2; Additional file 1: Table S3). MDMS7 is also enriched in gain19q13 and up-regulated interferon pathways. Both MDMS8 and MDMS9 have t $(14 ; 16)$ and $t(4 ; 14)$ patients, however, due to the low prevalence of $t(14 ; 16)$ patients in the study it does not appear to be the driver of any of these groups (Additional file 1: Fig. S3). MDMS8 is also significantly enriched in gain1q; del1p, del16q, del17p. In addition to $t(14 ; 16)$, MDMS9 shows a significant enrichment of gain1q, del13q14.3, del16q24.1, and mutations in ATM, DIS3, TP53 and TRAF3. MDMS10 is defined by del13q14.3 and mutations in DIS3 and PRKD2; while also presenting the highest significant enrichment for $\mathrm{t}(4 ; 14)$ and FGFR3 mutations compared to the other disease subgroups. The pattern of mutations in MDMS10 aligns with the activation of MEK/ERK signaling pathway [28]. MDMS11 presents down-regulation of interferon related pathways (in contrast to MDMS7) and reduced expression of $F B X W 2$ and KIF4B. MDMS12, mainly driven by $\mathrm{t}(11 ; 14)$, is also enriched in CCND1, IRF4 and NRAS mutations, over-expression of CCND1 and low expression of CCND2 (Figs. 1B and 2; Additional file 1: Table S3). 


\section{Identification and validation of MDMS8}

Survival analyses were performed to understand how the molecular disease subgroups relate to clinical outcome. Eleven of the disease subgroups share a progressionfree survival (PFS) and overall survival (OS) similar to standard risk patients (Fig. 3) [29]. In contrast, patients in MDMS8 display significantly poorer outcomes (median PFS, 19 months, log-rank $p<0.001$; median OS, 38 months, log-rank $p<1 \times 10^{-6}$ ) (Fig. 3). MDMS8 has enrichment for ISS III patients (Fisher exact test $p<0.05$ ) and biallelic TP53 (Fisher exact test $p<0.05$ ) (Fig. 2, Additional file 1: Tables S3 and S4). Moreover, among patients in MDMS8 carrying previously described highrisk markers in $\mathrm{MM}$, including $\mathrm{t}(4 ; 14), \mathrm{t}(14 ; 16)$, gain1q, del13q and del17p, both PFS and OS are significantly worse than among patients with similar genomic characteristics in non-MDMS8 clusters (Fig. 4). Two multivariate cox-regressions (one including clinical features and
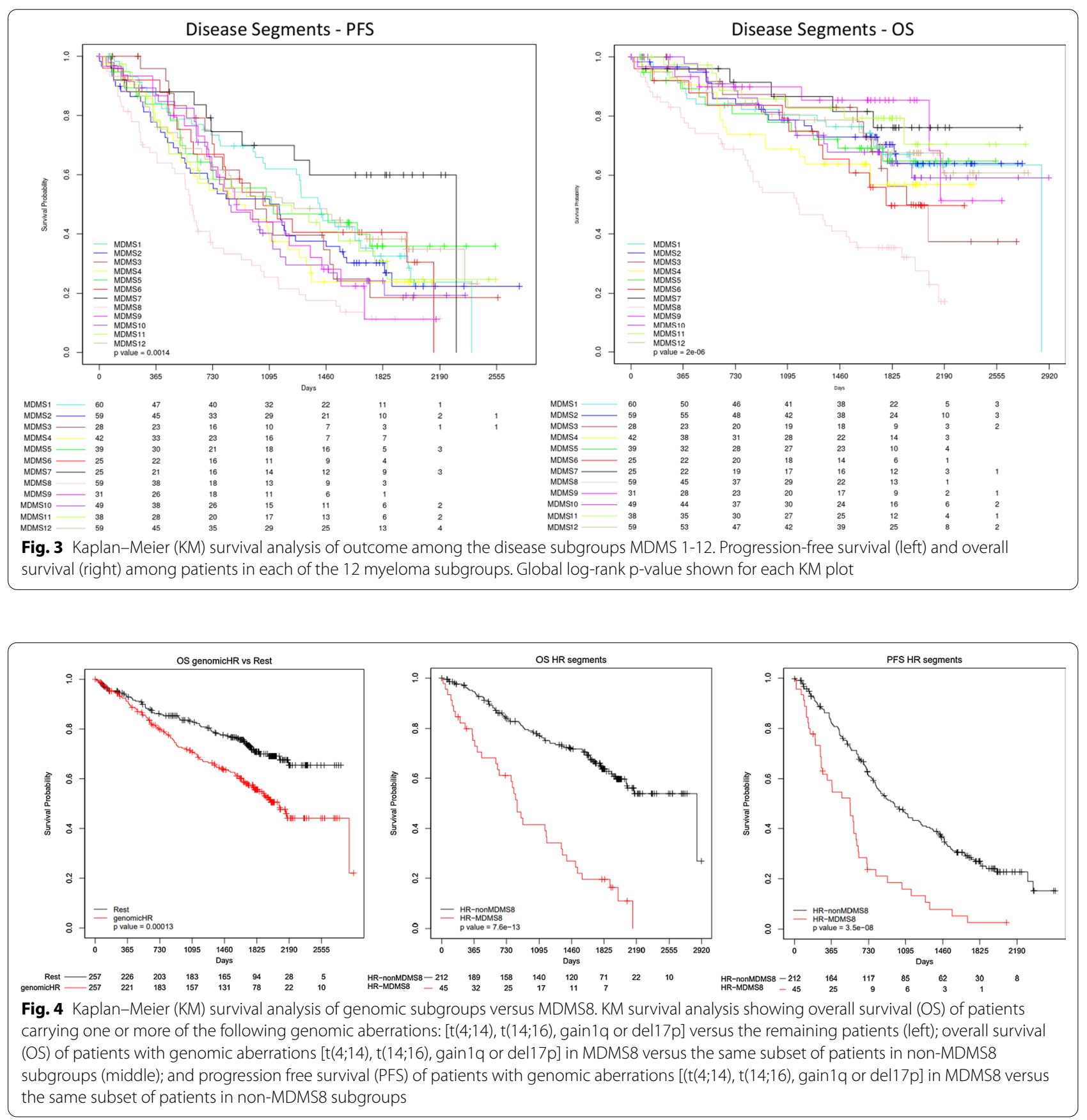
MDMS8 and the other including cytogenetic features and MDMS8) showed MDMS8 is an independent prognostic factor (Additional file 1: Fig. S4). Separate analyses for each of these high-risk markers, showed similar results, suggesting the presence of a common biology across these different genomic groups in addition to their high-risk features contribute to overall clinical outcome (Additional file 1: Fig. S5A-E).

In MDMS8 patients, DNA repair/damage related genes, such as ARID2, apoptosis related BIRC2, TRAF1, TRAF2 [30, 31], and genes associated with CDK function, including $M A X, R B 1$, and TP53 [32, 33], are significantly mutated. Differential GE analysis identified significant activation of genes controlling mitotic and DNA damage/ repair processes (CENPI, SKA1, NUF2, PLK1, AURKB, BIRC5 and BUB1), DNA synthesis (POLA1, PRIM1 and PRIM2), and checkpoints (MCM/CDC/RFC gene families and $C D K 1 / 2$ )-all generally involved in cell cycle related pathways (Fig. 5A). A differential gene expression analysis comparing patients with shared genomic characteristics (including $t(4 ; 14)$ or gain1q) in MDMS8 versus non-MDMS8 patients shows DNA repair, mitotic, checkpoint and $M Y C$ pathways significantly up regulated in MDMS8 (Additional file 1: Fig. S4A-B).
The genomes of MDMS8 samples present an increased loss of genes on various chromosomes, including 1 , 13, 14, 16 and 17 on the p arm (Fig. 1 and top panel of Fig. 5B) compared to the other molecular subgroups. We calculated the number of genomic cytobands containing a deletion and the total amount of genomic deletion in all samples (measured by the extent of deletion as percentage of the whole genome), which showed a significantly increased number of genomic regions having a loss in MDMS8 (median $>8 \%$ of genomic loss (Methods)) compared to the rest of the patients (median $<4 \%$ of genomic loss) ( $t$-test $p$-value $<1 \mathrm{e}-6$, bottom panel Fig. 5B). A gene set variant analysis (GSVA, see methods) on DNA damage/repair pathways (including REACTOME and DNA Damage Response (DDR) pathways [34]) showed a significant up-regulation of REACTOME DNA damage and repair pathways, as well as the DDR Homology-dependent recombination (HDR), Translesion Synthesis (TLS) and Base Excision Repair pathways in MDMS8 compared to the other NDMM patients (Fig. 5C, Additional file 1: Fig. S6).

To explore the prevalence of MDMS8 in other MM datasets, we built a GE classifier on the discovery data, applied it to independent cohorts (including IFM [5] and

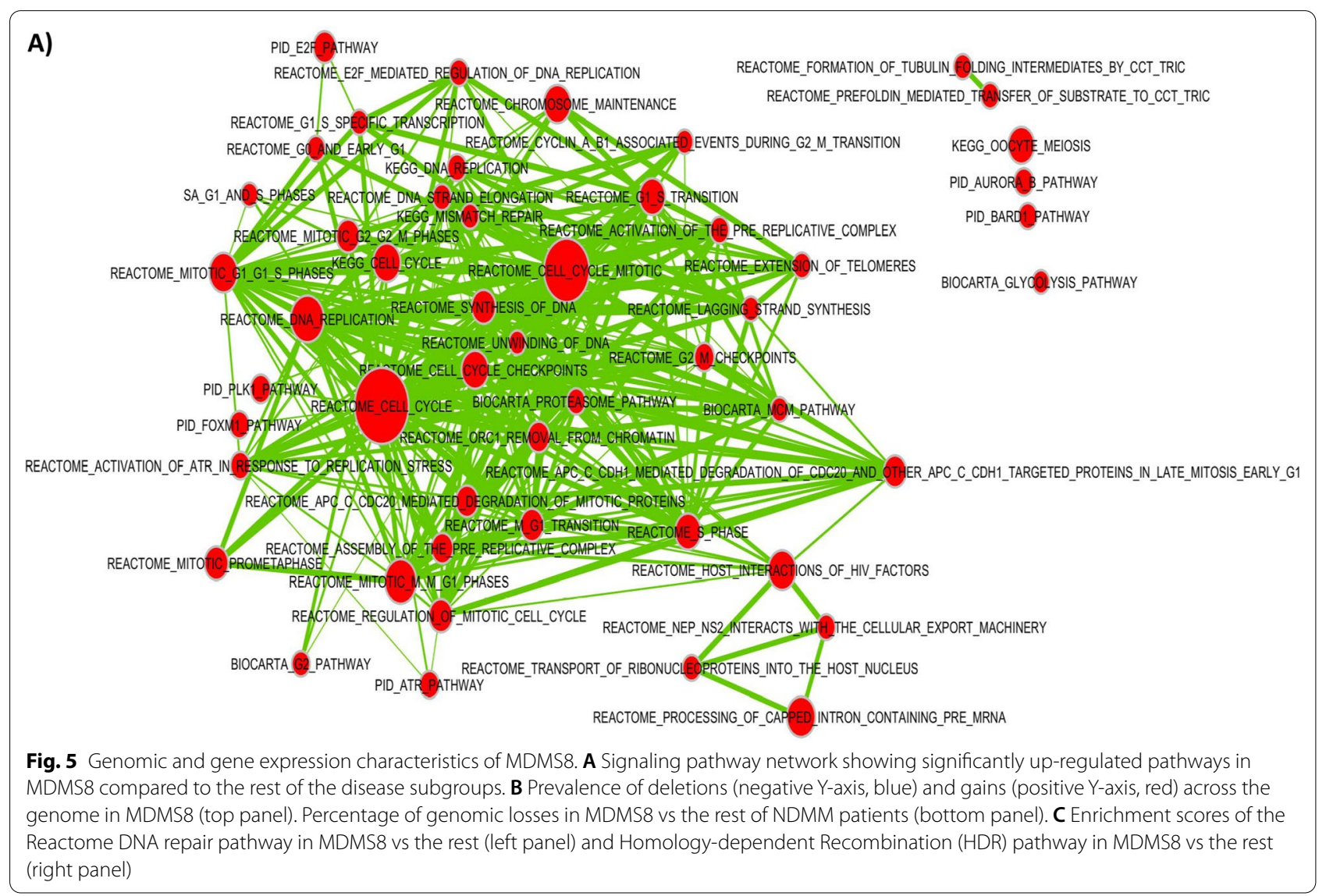




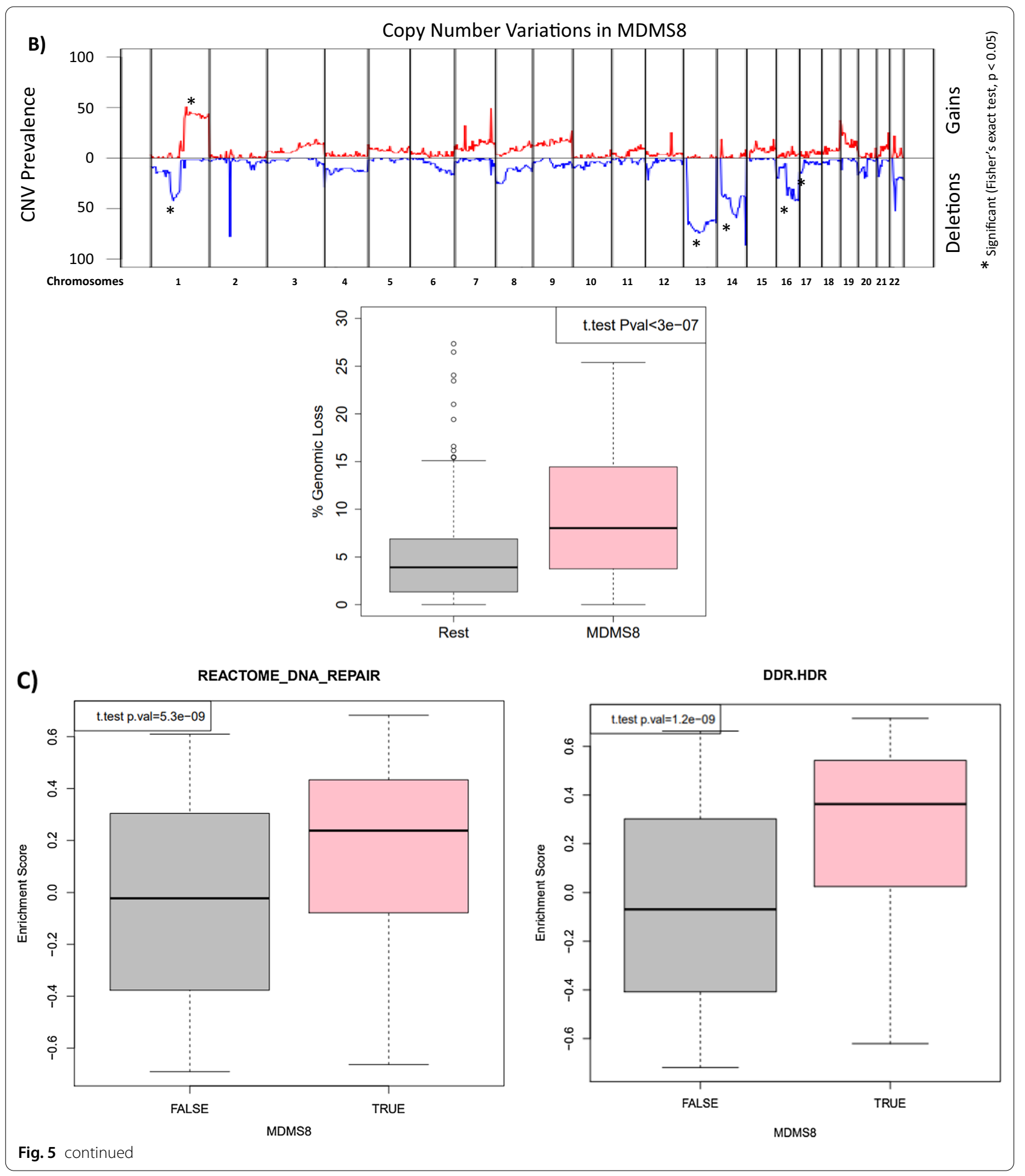

APEX [15, 35] (Additional file 1: Fig. 6A), and UAMS [17] (Fig. 6)), and explored prevalence and genomic properties (when available) of patients classified as 'MDMS8like' (Additional file 1: Fig. 6B). We generated a multiclass linear model classifier with lasso regression for feature selection based on gene expression, since it was the common datatype available across the datasets. The trained classifier comprised a linear model on the expression of 

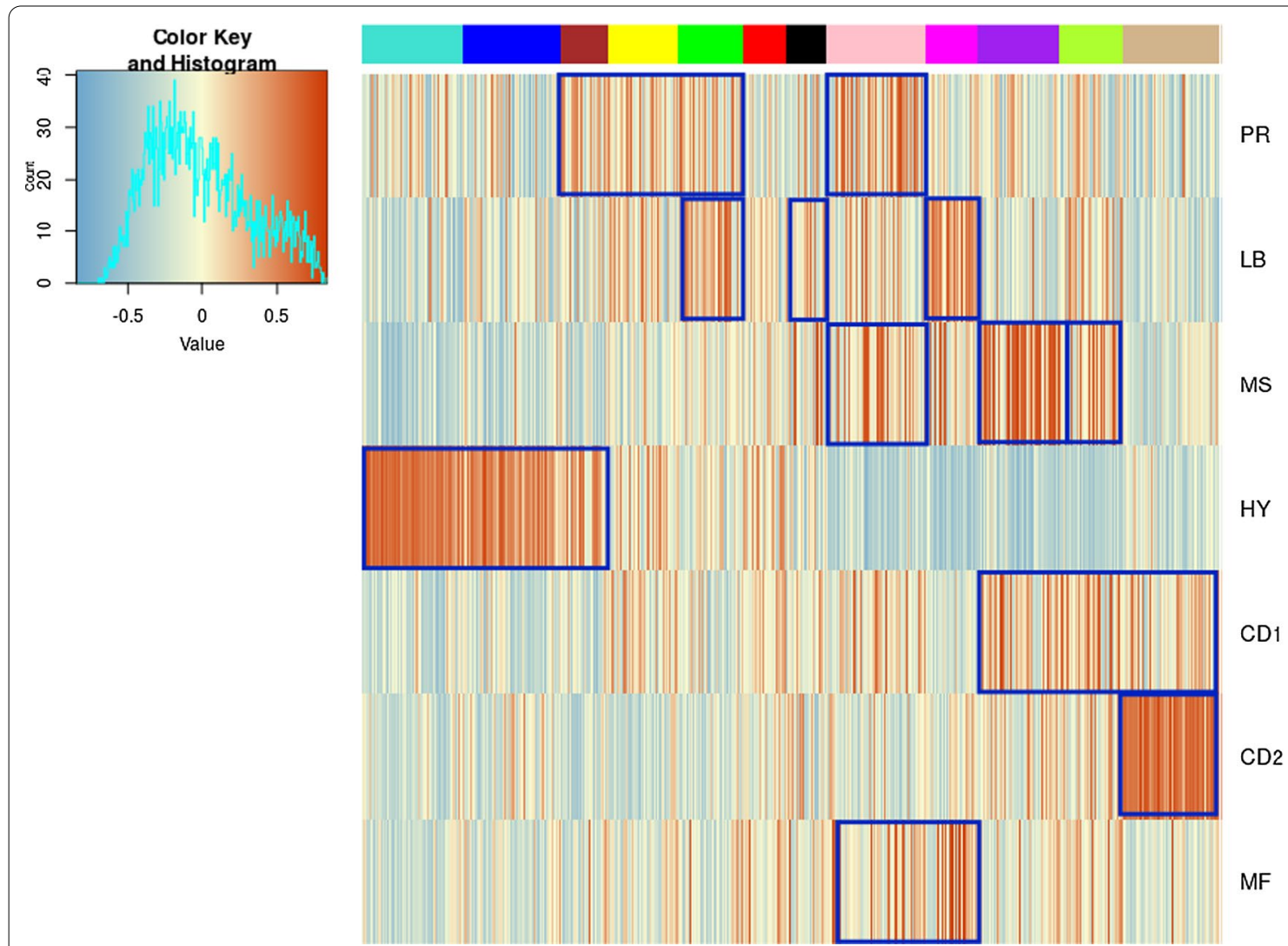

Fig. 6 Comparison of MDMS8 to other gene expression signatures. A gene expression enrichment of Zhan et al. GE patient subgroups signatures [15] across the twelve molecularly defined myeloma subgroups. Red represents positive enrichment; blue represents negative enrichment. Blue squares highlight significant association (enrichment scores t-test $p<0.01$ ) between the Zhan et al. signatures and MDMS disease subgroups

35 genes (Additional file 1: Table S4). The training performance of the classifier for MDMS8 has a recall $80 \%$ and precision of $75 \%$ (where false positives were mostly patients from MDMS9 and MDMS10) (Additional file 1: Table S5). Information on the training performance of the classifier for all clusters is shown in Additional file 1: Table S5, with a median recall of $60 \%$ and precision of 64\%; where most of the mis-classified calls happened between HY groups. Application of the classifier to the IFM dataset (Additional file 1: Table S3) identified a MDMS8-like group with similar prevalence $(\sim 12 \%)$ and significantly poorer OS (median OS not reached, long rank $p<1 \mathrm{e}-4$ ) (left panel of Additional file 1: Fig. S7A). Importantly, the MDMS8-like group in IFM also presented the high rate of genomic loss (median genomic loss MDMS8-like $>8 \%$ and rest $<4 \%$, Additional file 1 : Fig. S7B), validating not only the gene expression profile but also the genomic features. We applied the classifier to the APEX trial Affymetrix-based GEP dataset (RRMM) $[15,35]$, where, again, there was a significant difference in OS observed between MDMS8-like versus other RRMM patients (right panel of Additional file 1: Fig. S7A).
Prevalence of the MDMS8-like segment in the APEX trial was $<15 \%$. This analysis demonstrates that MDMS8like segment is reproducible across multiple datasets and that its poor OS is independent of treatment regimen.

\section{MDMS8 comparison to previously reported MM subgroups and high-risk signatures and biomarkers}

To place our analysis in the context of previous efforts, we explored similarities and differences between MDMS8 and other MM subgroups identified using GE datasets by Zhan et al. [15] and Broyl et al. [16]. In Fig. 6 (and Additional file 1: Fig. S8) MDMS8 shows a significant enrichment in the signature scores of the publicly described PR (proliferative), MS (MMSET) and MF (MAF) groups, which is coherent with MDMS8 since it contains $t(4 ; 14)$ patients (MS group), $t(14 ; 16)$ patients (MF group) and it shows dysregulation of cell cycle (PR group). Conversely, Zhan et al. groups are associated with multiple MDMS clusters, suggesting no 1:1 association between the two clustering approaches. We also applied our classifier to the Zhan et al. GEP discovery dataset and compared our cluster calls to theirs. This comparison, again, shows 
commonalities among some of the groups, such as the HY (hiperdiploid) from Zhan et al. which contains most of our MDMS3 and MDMS5, while CD2 maps uniquely to MDMS12; but it also shows clear differences, including MDMS4 (which from our genomics data is HY) which doesn't associate to the previously defined HY group. Also, MF and MS groups are subdivided into various MDMSs. Finally, MDMS8, presents a transversal profile to the previously defined GEP subgroups (containing patients from MF, MS, MY and PR) suggesting the biology of this group is more heterogeneous than what was previously described (Additional file 1: Table S6). While both attempts (Zhan et al. and ours) are unsupervised in nature, results show key differences between using GE only vs multi-omics integrative approach. Comparison of MDMS8 with the CNV clusters defined by Walker et al. [19] identifies significant enrichment of CN7 (characterized by gain1q and del13q); however, the CN7 cluster does not include all of the MDMS8 patients, notably excluding those with $\mathrm{t}(4 ; 14)$.

UAMS70 [3] and EMC92 [4] high-risk MM classifiers were applied to the discovery dataset to explore the overlap between patients deemed high-risk by these outcome-based classifiers and MDMS8 patients. MDMS8 captures a significant number of high-risk patients identified by both EMC92 (34\%) and UAMS70 (40\%). A third of MDMS8 patients, however, were not captured by these high-risk GE-classifiers (Additional file 1: Fig.
S9). Discordance among these groups is not unexpected, given that the number of shared genes between UAMS70 and EMC92 signatures is $<5 \%$. Moreover, unlike the GEclassifiers, the unsupervised approach used to identify MDMS8 was not based on clinical outcome.

\section{Master regulators drive transcriptional phenotype in MDMS8}

Finally, a master regulator (MR) analysis using msVIPER [36] was performed to elucidate the mechanisms linking genomic alterations to the transcriptional profiles of MDMS8. The master regulator genes were selected on the basis of impact on transcriptional changes of their inferred downstream targets (regulons) using a contextspecific gene regulatory model [37]. Ten MRs were identified (Fig. 7 and Additional file 1: Fig. S10), with seven of them showing positive activation in MDMS8: E2F2, a transcription factor member of the e2f family; $C K S 1 B$, a protein kinase regulator located in 1q21; $R B L 1$, which encodes a gene that is similar in sequence and possibly function to retinoblastoma 1 ( $R B 1$ ), significantly mutated in MDMS8; $P R K D C$, a protein kinase sensor for DNA damage incurred in DNA repair/recombination; RUSC1, related to the Trk receptor signaling mediated by the MAPK pathway; NUP93, described as tumor growth modulator via cell proliferation and actin cytoskeleton remodeling [38] and migration and invasion capacity of cancer cells [39], and MSN, Moesin, described as

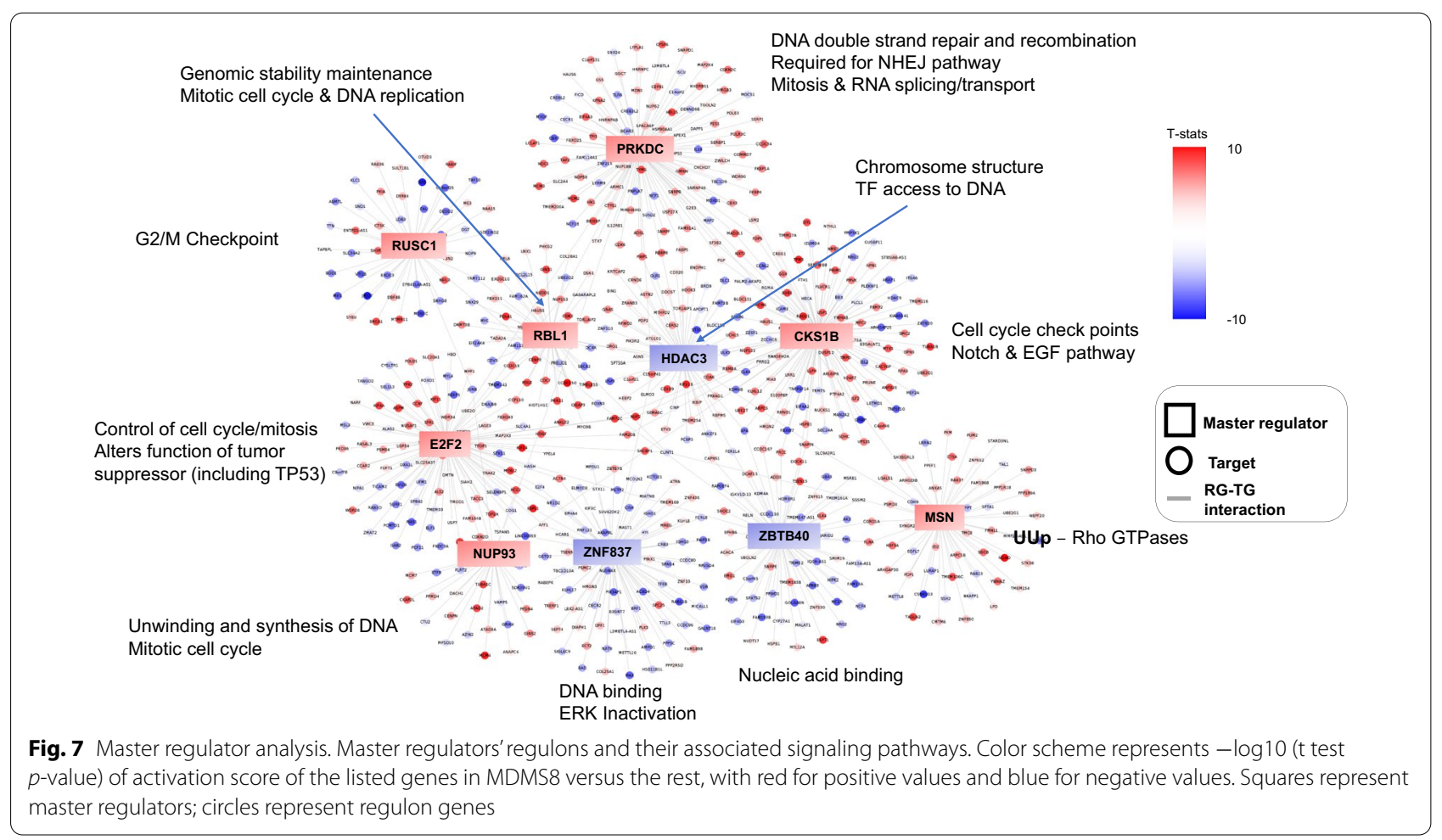


an unfavorable prognostic biomarker in various cancers [40-42]. Genes encoding the two zinc finger proteins (ZBTB40 and ZNF837) and the histone deacetylase 3 $(H D A C 3)$ were down-regulated MRs (Additional file 1: Fig. S10).

An enrichment analysis based on the regulons of MDMS8 MR was performed to understand MDMS8 biology and signaling functions controlled by these MRs. Most of the activated MRs control diverse biological processes (Fig. 7) including ones related to mitosis, such as the $E 2 F 2$ regulon, which contains the KIF family, and the $C K S 1 B$ regulon with $R A D 21$ and the $M C M$ family; or the $M S N$ regulon, associated with Rho GTPases (switches that regulate the actin cytoskeleton, influence cell polarity, microtubule dynamics, membrane transport pathways and transcription factor activity [43]). Cell cycle and DNA repair pathways in MDMS8 appear to be controlled by RBL1, NUP93 and PRKDC, although genes in the PRKDC regulon are involved also in spliceosome and RNA transport pathways, consistent with MDMS8 biology. Regulons downstream of the negatively activated MRs were not significantly associated with any specific signaling pathways, although they contained previously defined tumor suppressor genes, such as KDM4A [44] and E2F4 [45]. Of the MRs, the specific roles of PRKDC and RBL1 and their regulons in DNA damage/repair would be consistent with supporting the maintenance of MDMS8 myeloma cell's loss of genetic material.

\section{Discussion}

In this study, we describe molecular segmentation of NDMM by a joint modeling of multiple omics data types to identify common latent variables to group patient samples into biologically distinct disease subtypes. Our unsupervised analysis identifies twelve biological subgroups of MM, confirming hyperdiploidy-dependent and SV-dependent as the two predominant molecular subtypes of MM. Notably, we identified and replicated a new disease segment (MDMS8) that is enriched in diverse known high-risk genomic features, accompanied by various MM driver mutations and dysregulation of DNA damage and repair pathways and cell cycle/mitotic processes, alongside a genome loss, that had not been previously described in MM. Master regulator analyses identified potential drivers of the transcriptional program pointing to key pathways in DNA repair, cell proliferation, cell cycle progression and chromosomal stability and maintenance. PFS and OS are significantly inferior for patients in MDMS8 compared with patients in nonMDMS8 subgroups, even when patients in both cohorts carry the same high-risk genomic biomarkers, including 1q gain, del17p, $\mathrm{t}(4 ; 14)$ and/or $\mathrm{t}(14 ; 16)$. Our analysis shows for the first time that along with the different high risk markers (del17p, $\mathrm{t}(4 ; 14)$, amp1q) in NDMM there is a common transcriptional program linked to the accumulation of genome loss in a subset of those tumors. In our estimation, the identification of MDMS8 by the integration of multiple data-types enabled a transversal and improved molecular description of high risk MM biology over previous GE-based or $\mathrm{CN}$-based approaches. Not surprisingly, due to its association with poor clinical outcome, MDMS8 contains a significant number of patients picked up by gene expression based high-risk classifiers, EMC92 [4] and UAMS70 [3]. Besides, our integrated clustering analyses separate $\mathrm{t}(4 ; 14) \mathrm{MM}$ samples into multiple disease subgroups, including MDMS10 and MDMS8, all with high MMSET/NSD2 expression independent of the disease segment. The outcome and transcriptomic profile of MDMS8, however, are distinctly different from patients with $t(4 ; 14)$ in other disease subgroups, suggesting that overexpression of MMSET/NSD2 per se does not play a direct role in high-risk biology as had been previously discussed in the literature. While additional work is needed to tease out the implications of such observations, taken together, our results suggest that an integrated analysis of multiple data types could effectively sort out the heterogeneity of $t(4 ; 14)$ myeloma.

Identification of MDMS8, and its genomic loss linked with the dysregulated transcriptional phenotype prompted our exploration of functional drivers. The mechanism of the genome loss or its association with high-risk genetic loci is not clear at this time. Gene set enrichment analysis however revealed the relationship between MDMS8 transcription profiles with DNA repair/damage and cell cycle pathways, especially those directing the mitotic machinery and steps required for functional cell division. We envision that MDMS8 cells have adaptive mechanisms to tolerate excess DNA damage. It is likely that these transcriptional pathways are critical for repairing DNA damage as a consequence of DNA replication or induced to relieve the stress of multiple steps of proper chromosomal segregation during mitosis. All 7 MRs whose activities are up-regulated in MDMS8 are essential genes in MM, controlling key biological functions required for DNA repair/ damage, cell cycle check points for G1/S and G2/M, MYC-driven growth and survival pathways and mitotic processes. This analysis provides a pool of proteins to potentially target the underlying biological basis of the aggressive nature of the disease. Similar approaches in other cancers [24] have suggested possible synthetic lethal relationships between MRs which could provide novel combination approaches for therapeutics development in high-risk MM. These efforts could be combined or complemented with targeting the dysregulated DNA damage repair pathways. 
In conclusion, this work presents an integrative clustering-derived molecular classification of Multiple Myeloma using key genetic features with the transcriptome. We find a molecular segment enriched in extensive DNA loss, accompanied by upregulated DNA damage repair and cell cycle/mitotic pathways. This integrative analysis also illustrates that this type of approach could improve our understanding of the disease heterogeneity of Multiple Myeloma by studying the individual molecular segment such as MDMS8.

\section{Methods}

\section{Data processing}

\section{Gene expression}

RNA extraction, library preparation and sequencing for both MMRF CoMMpass and IFM/DFCI were previously described by Walker et al. [19] and https://research. themmrf.org.

\section{BAM to FastQ file conversion for MMRF CoMMpass cohort}

Previously aligned BAM files were collected from database of Genotypes and Phenotypes (dbGaP) and converted to FASTQ using Picard tools v2.1.1 to extract read sequences and base quality scores.

\section{Quantification}

FASTQ files from both cohorts were quantified using Salmon. Isoform level expressions were quantified with Quasi-mapping using GRCh38 cDNA reference genome from Gencode v24. Gene level abundances were calculated using tximport and isoform level TPM (transcript per million) estimates for each sample.

\section{Affymetrix gene expression}

GE from GSE2658 dataset hybridized to Affymetrix HG-U133 Plus 2 microarray was obtained using the RMA algorithm in the aroma.affymetrix framework (https://aroma-project.org/) based on a Brainarray reference file of the Ensembl 74 transcriptome to map probe IDs to gene symbols. Data for APEX trial was obtained from GSE9782 and the microarray used was Affymetrix UA133A/B array. Processing was based on MAS 5.0 normalization and $\log 2$ of MAS 5.0 intensities were utilized for analysis. Gene symbol annotation from Bioconducto (hg133 version 3.2.3) was used to map probe IDs to gene symbols.

\section{Scaling gene level expressions and selecting high variable genes}

GE was normalized for each sample against three housekeeping genes. 11 housekeeping genes [46] were originally tested and the top 3 genes with lowest standard deviation were selected. Geometric mean of these 3 housekeeping genes (NONO, PGK1 and VPS29) was used to scale gene level expressions.

\section{Calling copy number variants}

Preprocessing for copy number analysis has been described previously Walker et al. [19]. Genomic loss was calculated in each sample adding all the length of all the subgroups with a "loss" call from control-freec output (including both homozygous and heterozygous deletions). The final proportion of genomic loss is calculated per patient using size of genomic loss previously calculated over the genome size.

\section{SNV data}

SNVs were called and preprocessed as previously described [19]. After preprocessing, only missense mutations that were observed in $\geq 3 \%$ of the patients were kept for further analysis.

\section{SV data}

SVs were called and preprocessed as previously described [19]. Lowly prevalent SVs might be under-represented in our dataset due to size limitations.

\section{Clustering}

Two different clustering algorithms iCluster $+[26]$ and the Cluster of Clusters Algorithm (COCA) by the Cancer Genome Atlas Research Network [27] that integrate multiple OMICs data types with different approaches were run with a range of parameters to identify the combination which produced the most robust and stable clusters across our dataset. The number of clusters ranged between 2 and 20, and the optimal solution was selected based on Bayesian Information Criteria (BIC). Membership consistency across iterations was used to select iCluster + as the final clustering approach. More information can be found in the Additional file 2 .

\section{Biomarker analysis Differential gene expression}

Voom-LIMMA was run for GE analysis, using linear models to assess differential expression in the context of multifactor designed experiments [47]. It was implemented in the limma package for Bioconductor (http:// www.bioconductor.org) and applied to test differential relative abundance between conditions for each cluster independently. Significance p-values were corrected for multiple testing by the false-discovery method and deemed significant at an FDR threshold of 0.05 (5\%) [48].

\section{Pathway analysis}

Gene-set enrichment analysis (GSEA [49]) was applied to rank relative abundance ratios obtained 
during differential analysis for each comparison. Weighted enrichment statistic calculations were used instead of the classic unweighted ranking to account for fold change differences in addition to protein ranking. Gene categories assessed for enrichment corresponded to the canonical pathway collection (e.g. Reactome, Biocarta, KEGG) obtained from the MSigDB database (file: c2.cp.v5.2.symbols [50]). Enrichment p-values were corrected for multiple testing by FDR.

\section{Signature enrichment analysis}

GSVA $r$ package was used to calculate enrichment analysis of the various signatures. For UAMS70 [3] and EMC92 analysis, thresholds were refined to RNAseq data to select respectively $15 \%$ and $20 \%$ of the population with the highest scores.

\section{Identification of master regulators}

Master regulator analysis was performed using the msVIPER algorithm in the VIPER R package. More information can be found in the Additional file 2 .

\section{Classifier}

We utilized the glmnet package in CRAN (https://cran.rproject.org/web/packages/glmnet/index.html) to estimate a multinomial elastic net modelregression model with cross validation. The features were selected by estimating models with 100 nfolds on the top 3813 genes by coefficient of variance across all datasets. 42 genes identified across the cross-validation iterations were included in the final model. In order to make all the MM datasets comparable, they were normalized together with voom/ limma and dataset bias was removed with Combat $\mathrm{R}$ function [51]. Finally, all datasets were scaled independently by genes to median $=0$ and standard variation $=1$.

\section{Statistical analyses}

Various statistical tests from the stats v3.5.3 R [52] CRAN package were used to check significance of the association of the subgroups to different variables. Fisher's exact test for binary data (mutations/CNVs), t-test for continuous variables (GE pathway scores), and global log-rank test for outcome (PFS/OS).

\section{Supplementary Information}

The online version contains supplementary material available at https://doi. org/10.1186/s12920-021-01140-5.

Additional file 1. Supplementary document.

Additional file 2 . Cluster calls.
Acknowledgements

The authors acknowledge continued support for MGP from colleagues at BMS, especially Dorothy Fallows, Rupert Vessey, Douglas Bassett, Amit Agarwal and the Myeloma Disease Strategy Team.

\section{Authors' contributions}

The project was conceived and designed by AT. Funding acquisition by EF and AT. Project administration by MO, FT and EF. Oversight and management of resources (data generation, collection, transfer, infrastructure, data processing) by EF, FT and AT. Analyses and interpretation were designed and performed by $\mathrm{MO}, \mathrm{FT}, \mathrm{MT}, \mathrm{NS}, \mathrm{IJ}, \mathrm{KW}$ and AT. Data visualization performed by $\mathrm{MO}, \mathrm{IJ}$ and FT. Supervision and scientific direction provided by AT. The manuscript was written by MO, FT, EF and AT. All authors have read and approved the final manuscript.

Funding

Not applicable.

\section{Availability of data materials}

Sequencing data were deposited in the European Genome Archive under accession EGA00001001147 and EGA00001000036 or at database of Genotypes and Phenotypes (dbGAP) under accession phs000748.v5.p4.

Code availability

Our genomic pipeline code is provided under https://github.com/celgeneresearch/mgp_ngs. Methods used for analysis are publicly available.

\section{Declarations}

Ethics approval and consent to participate

Not applicable.

\section{Consent for publication}

Not applicable.

\section{Competing interests}

BMS Corporation: Employment, Equity Ownership: MO, FT, NS, IJ, KW, MT, EF, and AT. Funding for data processing and storage provided by BMS Corporation. No disclosures or competing interest relevant to this work for authors other than what is listed above.

\section{Author details}

${ }^{1}$ BMS Center for Innovation and Translational Research Europe (CITRE), A Bristol Myers Squibb Company, Sevilla, Spain. ${ }^{2}$ Bristol Myers Squibb, San Diego, CA, USA. ${ }^{3}$ Bristol Myers Squibb, 181 Passaic Ave, Summit, NJ 07901, USA.

Received: 21 September 2021 Accepted: 30 November 2021

Published online: 18 December 2021

\section{References}

1. Corre J, Munshi N, Avet-Loiseau H. Genetics of multiple myeloma: another heterogeneity level? Blood. 2015;125(12):1870-6.

2. Morgan GJ, Walker BA, Davies FE. The genetic architecture of multiple myeloma. Nat Rev Cancer. 2012;12(5):335-48.

3. Shaughnessy JD Jr, Zhan F, Burington BE, Huang Y, Colla S, Hanamura I, et al. A validated gene expression model of high-risk multiple myeloma is defined by deregulated expression of genes mapping to chromosome 1. Blood. 2007;109(6):2276-84

4. Kuiper R, Broyl A, de Knegt $Y$, van Vliet MH, van Beers EH, van der Holt $B$, et al. A gene expression signature for high-risk multiple myeloma. Leukemia. 2012;26(11):2406-13.

5. Magrangeas F, Nasser V, Avet-Loiseau H, Loriod B, Decaux O, Granjeaud $S$, et al. Gene expression profiling of multiple myeloma reveals molecular portraits in relation to the pathogenesis of the disease. Blood. 2003;101(12):4998-5006.

6. Chung TH, Mulligan G, Fonseca R, Chng WJ. A novel measure of chromosome instability can account for prognostic difference in multiple myeloma. PLoS ONE. 2013;8(6):e66361. 
7. Chng WJ, Braggio E, Mulligan G, Bryant B, Remstein E, Valdez R, et al. The centrosome index is a powerful prognostic marker in myeloma and identifies a cohort of patients that might benefit from aurora kinase inhibition. Blood. 2008;111(3):1603-9.

8. Hose D, Reme T, Hielscher T, Moreaux J, Messner T, Seckinger A, et al, Proliferation is a central independent prognostic factor and target for personalized and risk-adapted treatment in multiple myeloma. Haematologica. 2011;96(1):87-95.

9. Chng WJ, Chung TH, Kumar S, Usmani S, Munshi N, Avet-Loiseau H, et al. Gene signature combinations improve prognostic stratification of multiple myeloma patients. Leukemia. 2016;30(5):1071-8.

10. Samur MK, Minvielle S, Gulla A, Fulciniti M, Cleynen A, Aktas Samur A, et al. Long intergenic non-coding RNAs have an independent impact on survival in multiple myeloma. Leukemia. 2018;32(12):2626-35.

11. Walker BA, Mavrommatis K, Wardell CP, Ashby TC, Bauer M, Davies F, et al. A high-risk, Double-Hit, group of newly diagnosed myeloma identified by genomic analysis. Leukemia. 2019;33(1):159-70.

12. Thakurta A, Ortiz M, Blecua P, Towfic F, Corre J, Serbina NV, et al. High subclonal fraction of $17 \mathrm{p}$ deletion is associated with poor prognosis in multiple myeloma. Blood. 2019;133(11):1217-21.

13. Palumbo A, Avet-Loiseau H, Oliva S, Lokhorst HM, Goldschmidt H, Rosinol $\mathrm{L}$, et al. Revised international staging system for multiple myeloma: a report from international myeloma working group. J Clin Oncol. 2015:33(26):2863-9.

14. Greipp PR, San Miguel J, Durie BG, Crowley JJ, Barlogie B, Blade J, et al. International staging system for multiple myeloma. J Clin Oncol. 2005;23(15):3412-20.

15. Zhan F, Huang Y, Colla S, Stewart JP, Hanamura I, Gupta S, et al. The molecular classification of multiple myeloma. Blood. 2006;108(6):2020-8.

16. Broyl A, Hose D, Lokhorst H, de Knegt Y, Peeters J, Jauch A, et al. Gene expression profiling for molecular classification of multiple myeloma in newly diagnosed patients. Blood. 2010;116(14):2543-53.

17. Laganà A, Perumal D, Melnekoff D, Readhead B, Kidd BA, Leshchenko V, et al. Integrative network analysis identifies novel drivers of pathogenesis and progression in newly diagnosed multiple myeloma. Leukemia. 2018;32(1):120-30.

18. Hoang PH, Cornish AJ, Dobbins SE, Kaiser M, Houlston RS. Mutational processes contributing to the development of multiple myeloma. Blood Cancer J. 2019;9(8):60

19. Walker BA, Mavrommatis K, Wardell CP, Ashby TC, Bauer M, Davies FE, et al. Identification of novel mutational drivers reveals oncogene dependencies in multiple myeloma. Blood. 2018:132(6):587-97.

20. Berger AC, Korkut A, Kanchi RS, Hegde AM, Lenoir W, Liu W, et al. A comprehensive pan-cancer molecular study of gynecologic and breast cancers. Cancer Cell. 2018;33(4):690-705.

21. Robertson AG, Kim J, Al-Ahmadie H, Bellmunt J, Guo G, Cherniack AD, et al. Comprehensive molecular characterization of muscle-invasive bladder cancer. Cell. 2017;171(3):540-56.

22. Cancer Genome Atlas Research N, Linehan WM, Spellman PT, Ricketts CJ, Creighton CJ, Fei SS, et al. Comprehensive molecular characterization of papillary renal-cell carcinoma. New Engl J Med. 2016;374(2):135-45.

23. Papaemmanuil E, Gerstung M, Bullinger L, Gaidzik VI, Paschka P, Roberts $\mathrm{ND}$, et al. Genomic classification and prognosis in acute myeloid leukemia. N Engl J Med. 2016;374(23):2209-21.

24. Califano A, Alvarez MJ. The recurrent architecture of tumour initiation, progression and drug sensitivity. Nat Rev Cancer. 2017;17(2):116-30

25. Lim WK, Lyashenko E, Califano A. Master regulators used as breast cancer metastasis classifier. Pacific symposium on biocomputing pacific symposium on Biocomputing; 2009. p. 504-15

26. Mo Q, Wang S, Seshan VE, Olshen AB, Schultz N, Sander C, et al. Pattern discovery and cancer gene identification in integrated cancer genomic data. Proc Natl Acad Sci USA. 2013:1 10(11):4245-50.

27. Koboldt D, Fulton RS, McLellan MD, Schmidt H, Kalicki-Veizer J, McMichael JF, et al. Comprehensive molecular portraits of human breast tumours. Nature. 2012;490(7418):61-70.

28. Morgan GJ, He J, Tytarenko R, Patel P, Stephens OW, Zhong S, et al. Kinase domain activation through gene rearrangement in multiple myeloma. Leukemia. 2018:32(11):2435-44.

29. Binder M, Rajkumar SV, Ketterling RP, Dispenzieri A, Lacy MQ, Gertz MA, et al. Substratification of patients with newly diagnosed standard-risk multiple myeloma. Br J Haematol. 2019;185(2):254-60.
30. Wang CY, Mayo MW, Korneluk RG, Goeddel DV, Baldwin AS Jr. NFkappaB antiapoptosis: induction of TRAF1 and TRAF2 and C-IAP1 and c-IAP2 to suppress caspase-8 activation. Science (New York, NY). 1998;281(5383):1680-3.

31. Xiong Y, Ren YF, XU J, Yang DY, He XH, Luo JY, et al. Enhanced external counterpulsation inhibits endothelial apoptosis via modulation of BIRC2 and Apaf-1 genes in porcine hypercholesterolemia. Int J Cardiol. 2014;171(2):161-8.

32. Arcellana-Panlilio MY, Egeler RM, Ujack E, Magliocco A, Stuart GC, Robbins SM, et al. Evidence of a role for the INK4 family of cyclin-dependent kinase inhibitors in ovarian granulosa cell tumors. Genes Chromosomes Cancer. 2002;35(2):176-81.

33. Fiorentino FP, Tokgün E, Solé-Sánchez S, Giampaolo S, Tokgün O, Jauset T, et al. Growth suppression by MYC inhibition in small cell lung cancer cells with TP53 and RB1 inactivation. Oncotarget. 2016;7(21):31014-28.

34. Knijnenburg TA, Wang $L$, et al. Genomic and molecular landscape of DNA damage repair deficiency across the cancer genome atlas. Cell Rep. 2018;23(1):239-54.

35. Richardson PG, Sonneveld P, Schuster MW, Irwin D, Stadtmauer EA, Facon $T$, et al. Bortezomib or high-dose dexamethasone for relapsed multiple myeloma. N Engl J Med. 2005;352(24):2487-98.

36. Alvarez MJ, Shen Y, Giorgi FM, Lachmann A, Ding BB, Ye BH, et al. Functional characterization of somatic mutations in cancer using networkbased inference of protein activity. Nat Genet. 2016;48(8):838-47.

37. Alvarez MJ, Subramaniam PS, Tang LH, Grunn A, Aburi M, Rieckhof G, et al. A precision oncology approach to the pharmacological targeting of mechanistic dependencies in neuroendocrine tumors. Nat Genet. 2018;50(7):979-89.

38. Bersini S, Lytle NK, Schulte R, Huang L, Wahl GM, Hetzer MW. Nup93 regulates breast tumor growth by modulating cell proliferation and actin cytoskeleton remodeling. Life Sci Alliance. 2020;3(1):e201900623.

39. Ouyang X, Hao X, Liu S, Hu J, Hu L. Expression of Nup93 is associated with the proliferation, migration and invasion capacity of cervical cancer cells. Acta Biochim Biophys Sin. 2019;51(12):1276-85.

40. Barros FBA, Assao A, Garcia NG, Nonogaki S, Carvalho AL, Soares FA, et al. Moesin expression by tumor cells is an unfavorable prognostic biomarker for oral cancer. BMC Cancer. 2018;18(1):53.

41. Yu L, Zhao L, Wu H, Zhao H, Yu Z, He M, et al. Moesin is an independent prognostic marker for ER-positive breast cancer. Oncol Lett. 2019;17(2):1921-33.

42. Wang Q, Lu X, Wang J, Yang Z, Hoffman RM, Wu X. Moesin up-regulation is associated with enhanced tumor progression imaged non-invasively in an orthotopic mouse model of human glioblastoma. Anticancer Res. 2018;38(6):3267-72.

43. Etienne-Manneville S, Hall A. Rho GTPases in cell biology. Nature. 2002:420(6916):629-35.

44. Jin F, Kumar S, Dai Y. The lysine-specific demethylase KDM4A/JMJD2A acts as a tumor suppressor in multiple myeloma. Blood. 2018;132(1):191.

45. Feng Y, Li L, Du Y, Peng X, Chen F. E2F4 functions as a tumour suppressor in acute myeloid leukaemia via inhibition of the MAPK signalling pathway by binding to EZH2. J Cell Mol Med. 2020;24(3):2157-68.

46. Yang C, Pan H, Liu Y, Zhou X. Stably expressed housekeeping genes across developmental stages in the two-spotted spider mite, Tetranychus urticae. PLoS ONE. 2015;10(3):e0120833.

47. Law CW, Chen Y, Shi W, Smyth GK. Voom: precision weights unlock linear model analysis tools for RNA-seq read counts. Genome Biol. 2014;15(2):R29

48. Benjamini Y, Hochberg Y. Controlling the false discovery rate: a practical and powerful approach to multiple testing. J Roy Stat Soc Ser B (Methodol). 1995;57(1):289-300.

49. Subramanian A, Tamayo P, Mootha VK, Mukherjee S, Ebert BL, Gillette MA, et al. Gene set enrichment analysis: a knowledge-based approach for interpreting genome-wide expression profiles. Proc Natl Acad Sci USA. 2005:102(43):15545-50

50. Liberzon A, Subramanian A, Pinchback R, Thorvaldsdottir H, Tamayo P, Mesirov JP. Molecular signatures database (MSigDB) 3.0. Bioinformatics (Oxford, England). 2011;27(12):1739-40.

51. Leek JT, Johnson WE, Parker HS, Jaffe AE, Storey JD. The sva package for removing batch effects and other unwanted variation in high-throughput experiments. Bioinformatics (Oxford, England). 2012;28(6):882-3. 
52. Team RDC. R: a language and environment for statistical computing. $R$ Foundation for Statistical Computing. 2011;1:409.

\section{Publisher's Note}

Springer Nature remains neutral with regard to jurisdictional claims in published maps and institutional affiliations.

- fast, convenient online submission

- thorough peer review by experienced researchers in your field

- rapid publication on acceptance

- support for research data, including large and complex data types

- gold Open Access which fosters wider collaboration and increased citations

- maximum visibility for your research: over $100 \mathrm{M}$ website views per year

At BMC, research is always in progress.

Learn more biomedcentral.com/submissions 